www.jmscr.igmpublication.org

Impact Factor 5.244

Index Copernicus Value: 83.27

ISSN (e)-2347-176x ISSN (p) 2455-0450

crossref DOI:_http://dx.doi.org/10.18535/jmscr/v4i7.54

Journal Of Medical Science And Clinical Research

\title{
A Cross Sectional Study of Clinical Profile and Cardiac Manifstations in Patients with Primary and Secondary Dengue Fever in A Tertiary Care Hospital
}

\author{
Authors \\ Durga Krishnan ${ }^{1}$, Sarah Premraj ${ }^{2}$, Mayilananthi ${ }^{1}$, Vishwananth $\mathbf{C N}^{3}$, \\ Rajasekaran $\mathrm{D}^{4}$ \\ ${ }^{1}$ Associate Professor, ${ }^{2}$ Assistant Professor, ${ }^{3}$ Post graduate, ${ }^{4}$ Professor and Head,
}

Department of General medicine, Chettinad Hospital and Research Institute, Kelambakkam, Chennai

\begin{abstract}
Background: Dengue fever is one the most common mosquito borne viral disease. Cardiac system involvement is one of the atypical manifestations in dengue fever. So this study was conducted to find out cardiac involvement in patients Dengue fever. Also, the difference in hematological parameters in primary and secondary dengue fever was studied.

Materials and methodology: Patients admitted with dengue fever in Medicine Ward or Intensive Care Unit at Chettinad Hospital and Research institute, Kelambakkam were included in study from May 2014 to Feb 2015. Apart from routine laboratory investigations, these patients were subjected for cardiac evaluation in the form of ECG, Cardiac markers like Troponin I and ECHO.

Results: Total number of patients included in study was 60. Mean age group involvement was between $23.97 \pm 3.6$ years and males were more involved than females. Bleeding manifestations were seen in $4.5 \%$ of primary and $33.3 \%$ of secondary dengue patients, which was statistically significant $(p=0.006)$. Leucopenia was more in patients with primary infection, $63.6 \% \mathrm{vs} 22.2 \%$ in secondary $(p=0.005)$. With regard to thrombocytopenia, all the patients with secondary dengue had low platelets, as compared to $81.8 \%$ of primary Dengue $(p=0.04)$. Monocytosis was observed in both primary and secondary dengue fever. However, in primary dengue fever, monocytosis was found only in $33.3 \%$ of cases, whereas in secondary dengue fever it was seen in $66.7 \%(p=0.01)$. Bradycardia was the only significant cardiac manifestation found in $71.6 \%$ of patients.

Conclusion: In patients with dengue, fever with gastrointestinal symptoms and leucopenia are more likely to indicate primary infection, whereas bleeding manifestations with thrombocytopenia and monocytosis are more suggestive of secondary dengue. In our study, there was no significant cardiac involvement except bradycardia which reverted to normal after course of dengue illness.

Keywords: Dengue fever, Cardiac involvement, Leucopenia and Thrombocytopenia.
\end{abstract}

\section{Introduction}

Dengue is one of the most important mosquito borne illnesses worldwide. It is a caused by a flavivirus with four distinct serotypes (DENV1, DENV2, DENV3, and DENV4). The infection is ttransmitted by Aedes aegypti mosquitoes. Dengue infections can result in a wide spectrum of disease severity ranging from an influenza-like illness (dengue fever; DF) to the life-threatening dengue hemorrhagic fever (DHF)/dengue shock 
syndrome (DSS). In recent decades, the incidence of dengue infection has increased around the world and has become a major international public health concern. The disease is now endemic in more than 100 tropical and subtropical countries. The World Health Organization (WHO) estimates that there may be 50 million dengue infections worldwide every year ${ }^{1,2}$. Dengue fever can manifest as fever with bleeding tendencies, gastrointestinal symptoms, cardiac and neurological symptoms.

Infection with one serotype of DENV provides lifelong immunity to that serotype, but results only in partial and transient protection against subsequent infection by the other three serotypes. It is possible for a person to be infected as many as four times, once with each serotype. Serious disease is thought to occur mainly, though not exclusively, as a result of a second infection by a different serotype. It is well documented that sequential infection with different DENV serotypes increases the risk of developing DHF. Ninety percent of DHF infections occur in children less than 15 years of age. There is currently no specific treatment for DENV infection, although several potential vaccines are in development; therefore, the only method of preventing DENV transmission is vector (mosquito) control ${ }^{1,3}$.

The natural history of dengue infection usually follows a clear pattern. The majority of infections are asymptomatic and subclinical. Symptomatic disease follows an incubation period of four to seven days, and begins as an acute febrile illness with high temperature, malaise, retro-orbital headache, myalgia, backache, nausea, loss of appetite, and vomiting ${ }^{4}$.

For management purposes, clinical illness is divided into three phases: the febrile phase, the critical phase, and the recovery phase. Around the third to seventh day of illness, the critical phase, which is associated with a dropping platelet count, recovery of leukopenia, and defervescence, may begin. The critical phase is defined by the occurrence of features of plasma leakage: rising hematocrit, clinical or radiological evidence of third-space fluid leakage, and in some cases, hypotension.

A proportion of patients develop severe clinical shock, of which a minority proceed to develop relentless severe intractable shock, coagulopathy with bleeding, and multi-organ failure, which can culminate in death ${ }^{5}$.

Dengue fever can cause wide range of cardiac manifestations like myocarditis, pericarditis, conduction defect and atrial fibrillation, which are rare. Most of the cardiac manifestations are asymptomatic. Cardiac involvement is a poor prognostic feature.

\section{Aims and Objective}

1. To compare the clinical profiles of patients with primary and secondary Dengue fever

2. To find out the cardiac manifestations in patients with dengue.

3. To determine parameters for early detection of cardiac involvement in patients with dengue.

\section{Materials and methodology}

It's a Prospective Observational study conducted in Medicine Ward or Intensive Care Unit at chettinad hospital and research institute, Kelambakkam for 60 dengue fever cases.

\section{Inclusion Criteria}

- Patients serologically Dengue IgM alone, or IgM and IgG or NS1 antigen positive

- Patients aged above 18years

\section{Exclusion Criteria}

- Patients aged below 18years.

- Patients with congenital and acquired heart disease.

- Patients with chronic kidney disease.

- Patients not willing to be included in the study

\section{Sampling Frame}

- Patients admitted in Medicine Ward or Intensive Care Unit at chettinad hospital and research institute, Kelambakkam, TN, India over a period of three months. 


\section{Sample Size}

- $\operatorname{Sixty}(60)$

Patients admitted with dengue like fever were subjected to Complete Blood Count (CBC) and Dengue serology $\operatorname{IgM}$ and $\mathrm{IgG}$ or NS1 antigen. Those who were positive for Dengue serology IgM alone, or IgM and IgG or NS1 antigen were included in the study. Purpose of the study was explained to the patients and informed consent was taken. Patients were clinically examined and subsequently subjected to undergo ECG, Cardiac Markers and ECHO.

\section{Results}

Out of 60 dengue patients who were included in this study, mean age group was $23.97 \pm 3$.6. The youngest patient was 18 years old and the oldest patient was 42 years old. There were 54 (90\%) males and 6(10\%) females in our study. The baseline clinical manifestations are represented in Table 1. More than $70 \%$ of patients had fever at admission. Headache was found to be the commonest symptom, followed by myalgia and GI symptoms. Over $13 \%$ of the patients had bleeding manifestations during the course of hospital stay. On physical examination, $18.3 \%$ of the patients were found to have conjunctival suffusion and 8 patients had a rash (13.3\%). Vital sign examination revealed bradycardia in 43 patients $(71.6 \%)$. Hypotension was noted in 17 patients $(28.3 \%)$. Organomegaly in the form of hepatomegaly or splenomegaly or both was observed in $13(21.6 \%)$ of all the cases.
Out of 60 patients $54(90 \%)$ patients were positive for NS1 antigen, 28(46.6\%) patients were positive for IgM antibody and 18 patients were positive for $\mathrm{IgG}$ antibody. Primary dengue fever patients were $71 \%$ and secondary dengue fever patients were $29 \%$ respectively [Figure 1]. Out of these, $50 \%$ were found to be positive for NS1 antigen alone, indicating that they had primary Dengue fever detected early in the course of illness.

Based on the clinical features and laboratory investigations, the characteristics of patients with primary and secondary Dengue fever were compared, and the results are presented in Table 2. Fever was there in $72.7 \%$ of primary dengue patients compared to $55.6 \%$ in secondary dengue patients. Bleeding manifestations were seen in $4.5 \%$ of primary and $33.3 \%$ of secondary dengue patients, which was statistically significant $(\mathrm{p}=0.006)$. Leucopenia was more $(63.6 \%)$ in patients with primary infection vs $22.2 \%$ in secondary $(p=0.005)$ [Figure 2]. Looking at thrombocytopenia, all the patients with secondary dengue had low platelets, as compared to $81.8 \%$ of primary Dengue $(p=0.04)$ [Figure 3]. Monocytosis was observed in both primary and secondary dengue fever. However, in primary dengue fever, monocytosis was found only in $33.3 \%$ of cases, whereas in secondary dengue fever it was seen in $66.7 \%(\mathrm{p}=0.01)$ [Figure 4].

Cardiac markers like Troponin I and CKMB were negative in all the primary and secondary dengue fever patients. ECG was also normal except sinus bradycardia was observed in $71.6 \%$ patients. ECHO was also normal in all the patients.

Table 1: Baseline characteristics of Dengue fever patients.

\begin{tabular}{|l|l|}
\hline Clinical manifestation & No. Of cases (\%) \\
\hline Headache & $20(33.3)$ \\
\hline Gastrointestinal symptoms & $12(20)$ \\
\hline Severe myalgia & $18(30)$ \\
\hline Bleeding manifestations & $8(13.3)$ \\
\hline Rash & $4(6.6)$ \\
\hline Organomegaly (liver/ spleen enlargement) & $13(21.6)$ \\
\hline
\end{tabular}




\section{JMSCR Vol||04||Issue||07||Page 11515-11521||July}

Table 2: Comparison of clinical and hematological profile of primary and secondary dengue

\begin{tabular}{|l|c|c|c|}
\hline Parameter & Primary dengue & Secondary Dengue & P value \\
\hline Fever & $72.7 \%$ & $55.6 \%$ & 0.18 \\
\hline Bleeding & $4.5 \%$ & $33.3 \%$ & 0.006 \\
\hline Leucopenia & $63.6 \%$ & $22.2 \%$ & 0.005 \\
\hline Thrombocytopenia & $81.8 \%$ & $100 \%$ & 0.04 \\
\hline Monocytosis & $33.6 \%$ & $66.7 \%$ & 0.01 \\
\hline
\end{tabular}

Figure 1: Primary and secondary dengue fever patients.

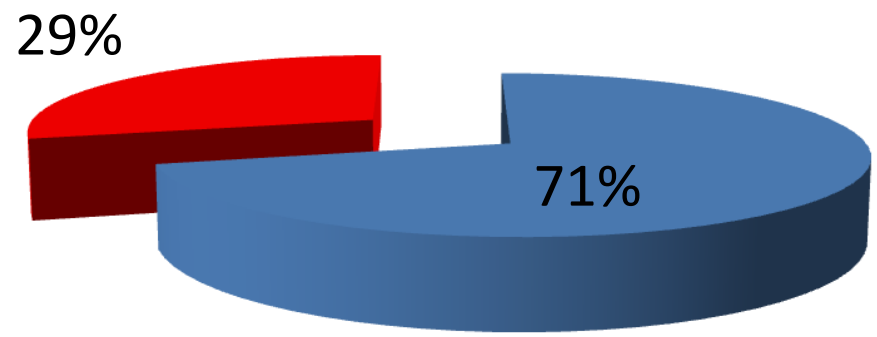

Primary

Secondary

Figure 2: Leucopenia in primary and secondary dengue fever patients.

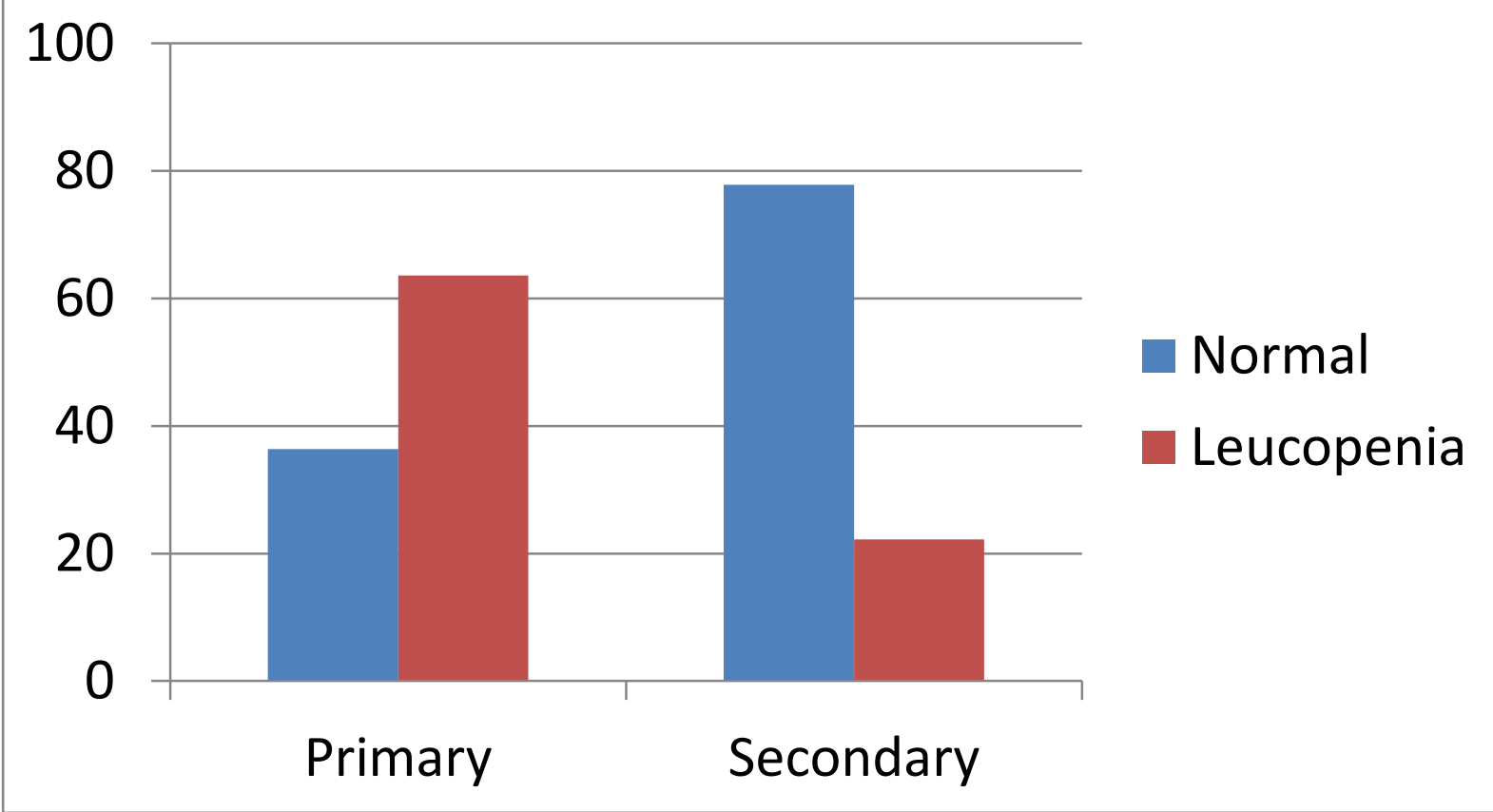


Figure 3: Thrombocytopenia in primary and secondary dengue fever patients.

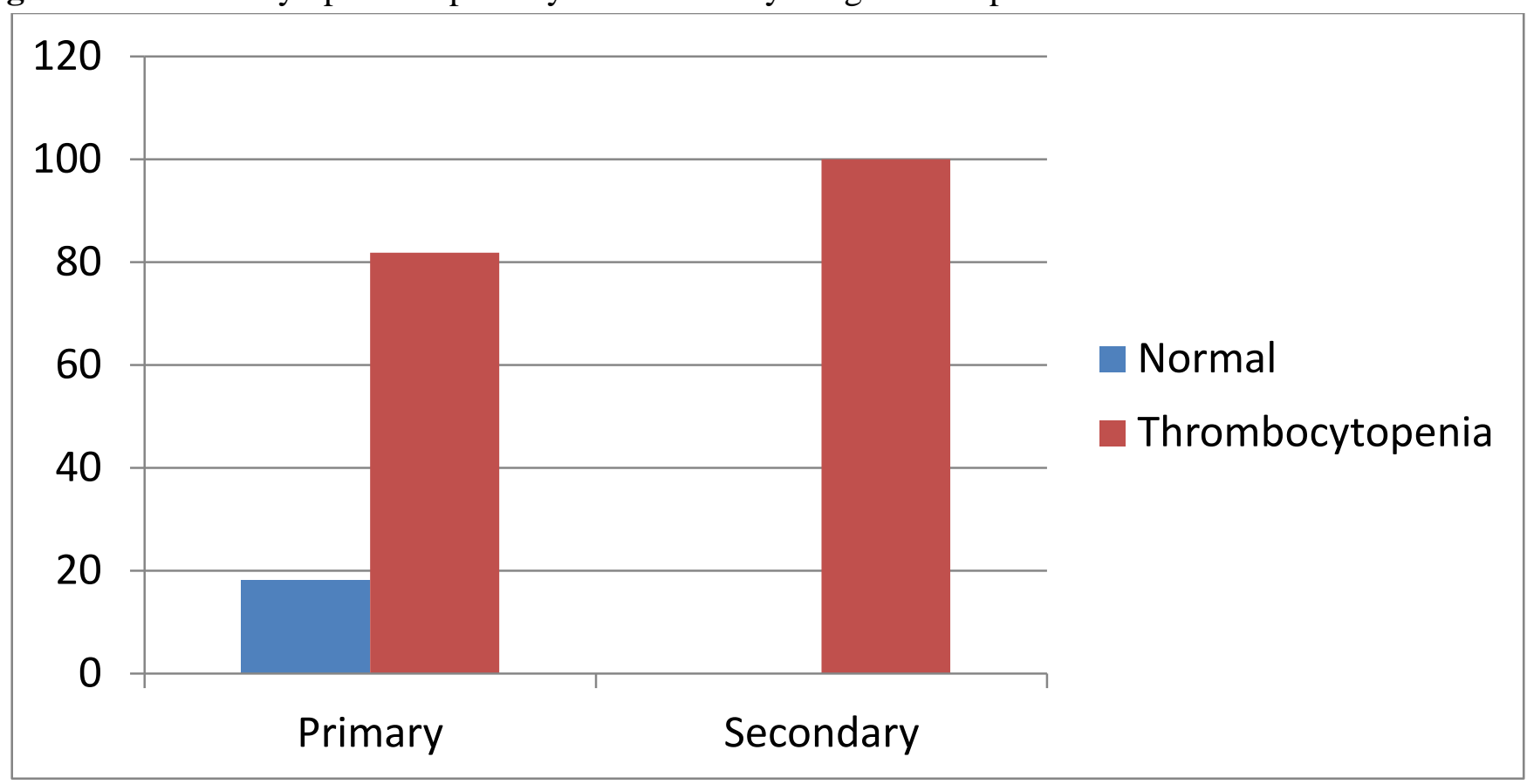

Figure 4: Monocytosis in primary and secondary dengue fever patients

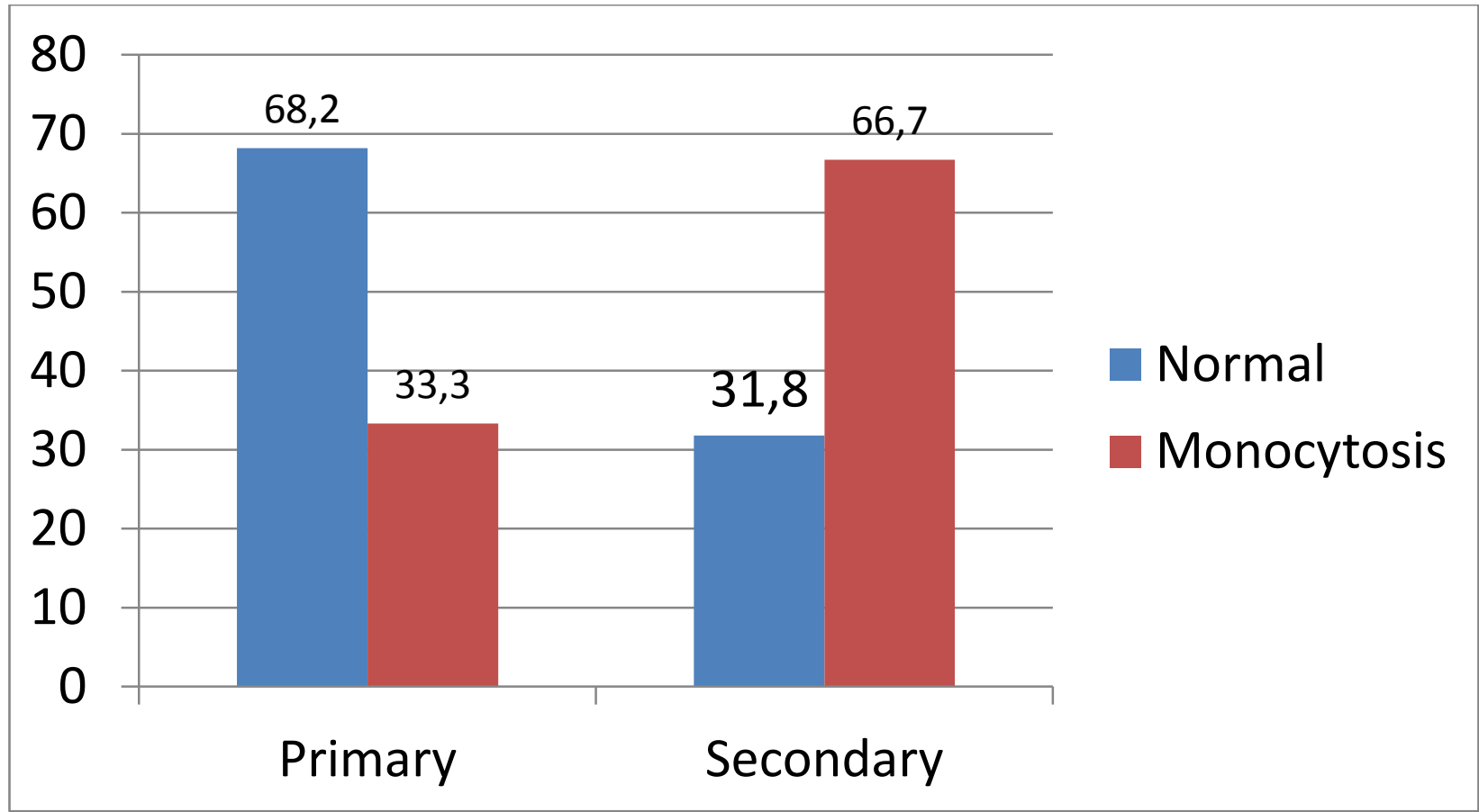

\section{Discussion}

In our study males were more involved than females. Primary dengue patients presented more commonly with fever and gastrointestinal manifestation than secondary dengue patients. Secondary dengue fever patients presented with more bleeding manifestations. Leucopenia was more seen in primary dengue patients. Thrombocytopenia was more in secondary dengue patients than primary. Monocytosis was present in both primary and secondary dengue patients.

In the present study, comparison was made between the hematological parameters in primary and secondary infections. We aimed to establish whether it is possible to differentiate between these two based on hematological indices. To the best of our knowledge, such data are not available so far in our population. Based on the results 
obtained in our study, we can postulate that in patients with primary dengue infection, there is more incidence of fever and gastrointestinal symptoms, along with leucopenia. In comparison, secondary infection with Dengue was more likely to present with bleeding manifestations, and laboratory evidence of thrombocytopenia and monocytosis.

We also studied the cardiac manifestations in dengue fever. In our study, ECG showed only transient rate abnormality in the form of bradycardia, there were no other changes. Cardiac markers like Troponin I and CKMB were negative in both primary and secondary dengue fever patients. ECHO was normal in both group of patients.

Several case reports and studies have been done on cardiac involvement in dengue fever. Some of case reports and studies showed that there is significant cardiac involvement in dengue fever but few studies also showed no cardiac involvement.

Dominic Wichmann et al studied cardiac involvement in dengue virus infections during the 2004/2005 epidemic in Srilanka, and concluded that there was elevated cardiac enzymes in both primary and secondary dengue infection ${ }^{6}$. Carlos Henrique Miranda stated that the heart can be affected during dengue virus infection in a considerable percentage of patients needing hospital admission. Clinical manifestations range from mild elevation of biomarkers to myocarditis and/or pericarditis. These cardiac complications might be underdiagnosed in clinical practice and can contribute to the mortality observed in dengue $^{7}$.Ravindra $\mathrm{L}$ et al established that cardiac involvement in dengue fever runs a benign course without any long term complications ${ }^{8}$.

In the Indian scenario, Vishal Kumar Gupta et al reported that myocardial involvement was subclinical as 2D-Echo was normal in patients ${ }^{9}$. Sheetal et al concluded that the most common cardiac manifestations noted were transient rhythm abnormalities, of which sinus bradycardia was the commonest. There was no evidence of myocarditis in any of their patients ${ }^{10}$. Jitendra Ingole et al also found that relative bradycardia was the only cardiac manifestation found in dengue fever patients ${ }^{11}$.

The limitations of our study include the relatively younger age group of patients in the study. This may be the reason for the low incidence of cardiac abnormalities. Also, the population studied included predominantly migrant labourers and may not be representative of the general population. Further studies may be needed including patients across all age groups and socioeconomic strata to clearly establish the relationship between dengue and cardiovascular events.

\section{Conclusion}

In patients with dengue, fever with gastrointestinal symptoms and leucopenia are more likely to indicate primary infection, whereas bleeding manifestations with thrombocytopenia and monocytosis are more suggestive of secondary dengue. In our study, there was no significant cardiac involvement except bradycardia which reverted to normal after course of dengue illness.

\section{References}

1. WHO Fact sheet $N^{\circ}$ 117: Dengue and dengue haemorrhagic fever. (2008) Available: http://www.who.int/mediacentre/factsheets/fs 117/en/

2. Dengue and Dengue Hemorrhagic Fever: Information for Health Care Practitioners CDC Division of Vector-Borne Infectious Diseases.

3. World Health Organization (2009) Dengue: Guidelines for Diagnosis, Treatment, Prevention \& Control.

4. Malavige G, Fernando S, Fernando D, Seneviratne S. Dengue viral infections. Postgraduate Medical Journal. 2004;80(948):588-601.

5. Sam SS, Omar SFS, Teoh BT, Abd-Jamil J, AbuBakar S. Review of Dengue Hemorrhagic Fever Fatal Cases Seen 
Among Adults: A Retrospective Study. Farrar J, ed. PLoS Neglected Tropical Diseases. 2013;7(5):e2194.

6. Wichmann D1, Kularatne S, Ehrhardt S, Wijesinghe S, Brattig NW, Abel $\mathrm{W}$, Burchard GD. Cardiac involvement in dengue virus infections during the 2004/2005 dengue fever season in Sri Lanka. Southeast Asian J Trop Med Public Health. 2009 Jul;40(4):727-30.

7. Miranda $\mathrm{CH} 1$, Borges Mde C, Matsuno AK, Vilar FC, Gali LG, Volpe GJ et al. Evaluation of cardiac involvement during dengue viral infection. Clin Infect Dis. 2013 Sep;57(6):812-9.

8. Satarasinghe R. Asymptomatic Myocardial Involvement In Acute Dengue Virus Infection in A Cohort Of Adult Sri Lankans Admitted To A Tertiary Referral Centre. Br J Cardiol. 2007;14:171-3.

9. Gupta V. Subclinical Cardiac Involvement in Dengue Haemorrhagic Fever. Journal, Indian Academy of Clinical Medicine 2010; 11(2): 107-11.

10. Sheetal S, Elizabeth J. A Study on the Cardiac Manifestations of Dengue. Journal of The Association of Physicians of India. 2016; 64: 30-34.

11. Ingole J. Relative Bradycardia in Dengue Fever. Indian Journal Of Applied Research. 2015; 5(1): 424-425. 